\title{
Función informacional de las escisiones en español ${ }^{1}$
}

\author{
Viviana Núñez Alvarado² \\ Universidad Nacional, Costa Rica
}

\begin{abstract}
resumen
Ha sido frecuente que el español de Centroamérica como variante se ha excluido de los estudios lingüísticos, tanto de corte variacionista como teóricos, y muchos de sus aspectos estructurales no han sido abordados. En este estudio se analiza una de las estructuras con función informacional en el español de Centroamérica, las oraciones escindidas, y se concluye que tienen características propias que no concuerdan con las descripciones existentes.
\end{abstract}

\begin{abstract}
The Spanish of Central America is a variant which has frequently been excluded from both variationist and theoretical studies in linguistics. Many of its structural aspects have not been covered. In this study one of these structures with an informational function in the Spanish of Central America is addressed: cleft sentences. It is concluded that they have characteristics which do not coincide with existing descriptions.
\end{abstract}

Palabras clave: estructura informacional, oraciones escindidas, español de Centroamérica

Keywords: information structure, cleft sentences, Spanish in Central America

1 Versión revisada de la ponencia leída en el marco del I Simposio Internacional del Programa de Lingüística Centroamericana (PROLINCA) «Centroamérica: un microcosmo lingüístico, llevado a cabo los 7 y 8 de mayo 2012, en la ciudad de Heredia, en el campus Omar Dengo de la Universidad Nacional de Costa Rica. Recibido: 5 de febrero de 2012; aceptado: 28 de junio de 2012.

2 Escuela de Literatura y Ciencias del Lenguaje. Correo electrónico: vinuneza@gmail.com

$$
L_{\text {etras }} 51 \text { (2012), ISSN 1409-424X }
$$




\section{Introducción}

En lingüística moderna se entiende por estructura de la información el componente de la gramática oracional en el cual las proposiciones, en tanto representaciones de estados de cosas, se parean con estructuras léxicogramaticales, de acuerdo con el estado mental de los interlocutores que usan e interpretan esas estructuras, como unidades de información, en determinados contextos discursivos ${ }^{3}$. La definición Lambrecht remite a ejemplos en inglés y en francés, pero no hace referencia al español. El objetivo de este trabajo es analizar, desde la perspectiva de Lambrecht, la estructura informacional en el español, en particular el español de Centroamérica.

A inicios del siglo xx, algunos de los temas tratados por Lambrecht, sobre todo el orden de la oración y la entonación, se estudiaron en el contexto de la relación entre gramática y psicología (Teoría de Poll, 1909). Este tipo de estudio fue denominado perspectiva funcional de la oración, por académicos de la Escuela de Praga, información de la estructura o tema, por Halliday, paquetes de información, por Chase; discursos pragmáticos, y más recientemente, informáticos, por Valdubi. Son términos diferentes para referirse a la estructura de la información. Según Lambrecht ${ }^{4}$, lo que hace incompletos esos acercamientos es que no contemplan la idea de que ciertas propiedades formales de la oración no pueden entenderse sin considerar los contextos lingüísticos y extralingüísticos, en los cuales la oración adquiere las propiedades correspondientes. Es decir, la estructura de la información se refiere a la forma como la oración es relacionada con estados mentales de los hablantes y los oyentes. Según el Centro Virtual Cervantes, algunos lingüistas de la Escuela de Praga a mediados del siglo xx, empezaron a realizar investigaciones sobre la estructura de la información; primero, aplicándola

\footnotetext{
3 Knud Lambrecht, Information Structure and Sentence Form (Nueva York: Cambridge University Press, 1994) 5.

4 Lambrecht.
} 
en la oración y más adelante en textos. Segmentaron la perspectiva funcional de la oración en dos partes: tema (información conocida) y rema (información nueva) ${ }^{5}$.

Lambrecht se centra en la relación entre la estructura de las oraciones y los contextos lingüísticos y extralingüísticos en los cuales las oraciones son usadas. Se refiere al sistema de opciones que las gramáticas ofrecen a los hablantes para expresar ciertos contenidos en diferentes formas gramaticales bajo diferentes circunstancias del discurso. En su estudio, Lambrecht propone el término de alo-ración para referirse al hecho de que estructura básica tiene diferentes alternativas para dar a entender lo mismo; por ejemplo, la oración (1): el hablante tiene las siguientes alternativas para expresarla:

(1a) Bolivar envenenó al perro. (oración no marcada)

(1b) El perro fue envenenado por Bolívar. (oración pasiva)

(1c) Envenenaron al perro. (oración impersonal)

(1d) El que envenenó al perro fue Bolívar. (oración pseudo escindida) (1e) Fue Bolívar el que envenenó al perro. (oración escindida)

Detengámonos en una de esas variantes: las oraciones escindidas. Se examina, por una parte, si lo dicho en relación con la estructura informacional de las oraciones escindidas se manifiesta en el español de Centroamérica, y, por otra, detectar posibles funciones divergentes. El análisis se funda en una base de datos empírica. Los datos provienen de un corpus titulado El español de Centroamérica: materiales para su estudio, el cual incluye los datos aportados por una muestra de seis informantes por país seleccionados bajo parámetros específicos - de cada una de las ciudades capitales de Centroamérica.

5 Centro Virtual Cervantes, Progresión temática. Disponible en: http://cvc.cervantes.es (consultado el 5 de noviembre de 2011). 


\section{Estructura interna de las oraciones escindidas en español}

Como se ha señalado, la escisión consiste en separar el constituyente focalizado ${ }^{6}$ del resto de la oración, mediante el copulativo ser, como en (2):

(2) Fue Paco el que vino ayer.

Lehmann ${ }^{7}$ propone para la oración escindida la siguiente estructura: $\left.[\mathrm{X}]_{\text {foco }}[\mathrm{Y}]_{\text {O.extrafoc }}\right]$. Se llama oración escindida porque consta de las dos partes X y Y, en comparación con una oración simple. Considérese la relación entre (1a) y (1e), repetida aquí como (3a) y (3b):

(3a) Bolívar envenenó al perro. (oración simple)

(3b) Fue Bolívar el que envenenó al perro. (oración escindida)

El significado de una oración escindida $[\mathrm{XY}]$ es el siguiente: 'Entre todos los elementos que podrían desempeñar la misma función en $Y$, no es ningún otro que $X^{\text {s. }}$. Es decir: el significado de $\mathrm{Y}$ se presupone, y ante ello se destaca X. Según Lambrecht, en francés se utilizan mucho estas oraciones escindidas para hacer hincapié, mientras que en inglés, la proposición expresada en las cláusulas relativas de las construcciones escindidas debe ser pragmáticamente presupuesta, aunque se permite una acomodación pragmática.

En lingüística hispánica a este tipo de oraciones se los denomina de muy diversa manera: perífrasis de relativo, fórmulas

6 «El foco de una oración es el elemento sintáctico al que se le da prominencia mediante entonación, un orden especial que lo destaque (usualmente al inicio) u otro procedimiento». Tomado de: Christian Lehmann, Oración escindida. En web: www.christianlehmann.eu (consultado el 5 de noviembre de 2011).

7 Lehmann (consultado el 5 de noviembre de 2011).

8 Lehmann (consultado el 5 de noviembre de 2011). 
perifrásticas de relativo, oración hendida, etc. Según Moreno Cabrera $^{9}(1983)$, «se pueden convertir las oraciones españolas en PdR (perífrasis de relativo) separando o escindiendo un constituyente, poniendo en su lugar un pronombre relativo y luego añadiendo el constituyente escindido al principio, presidido o seguido de la cópula o al final, precedido de la copula. Las oraciones españolas que empiezan por la clausula que contiene el pronombre relativo se llaman oraciones pseudo-escindidas; las que comienzan por la cópula son oraciones escindidas», como en (4a) y (4b):

(4a) Elque descompuso el radio fue Gabino. (oración pseudoescindida) (4b) Fue Gabino el que descompuso el radio. (oración escindida)

Un repaso de la bibliografía disponible sobre el tema muestra que la función informacional de estas construcciones escindidas no se ha estudiado en detalle. Las investigaciones se centran, sobre todo, en las oraciones pseudoescindidas; por ejemplo, en Castellani (1977); Guitart (1989); Moreno Cabrera (1999); Sedano (1990, 1995, 2001 y 2003); Toribio (1992); Acevedo y otros (1996); Bosque (1999); y Lavachi (2001). Fernández ${ }^{10}$ reconoce, aparte de la estructura canónica propuesta por Lehmann ${ }^{11}$, otra forma de la oración escindida, que ella califica como «más improductiva», que presenta el constituyente focalizado en primer lugar, como en (5):

(5) Ana fue la que vino ayer.

Parte de diferenciar las oraciones escindidas de las atributivas. Para Fernández, las escindidas son la forma más eficaz de expresar

9 Moreno Cabrera, «Las funciones informativas. Las perífrasis de relativo», en Ignacio Bosque y Violeta Demonte (Eds), Gramática descriptiva de la lengua española (Madrid: Espasa Calpe, 1999).

10 María Jesús Fernández, «Sobre formas de ambigüedad de las oraciones escindidas: sintaxis y discurso», Revista Estudios de Lingüística de la Universidad de Alicante 15 (2001):285-305.

11 Lehmann (consultado el 5 de noviembre de 2011). 
relevancia y se usan para enfatizar e identificar, mientras que las atributivas son predictivas, especificativas y atributivas.

Gutiérrez $^{12}$ (2008) analiza la función de los tópicos y los focos en las oraciones. Entiende el foco como el elemento sintáctico al que se le da prominencia mediante entonación u orden que lo destaque. Distingue entre foco contrastivo (típico de oraciones escindidas) y foco informativo, elemento que coincide frecuentemente con información nueva, mientras que el tópico o tema que es «de lo que se habla en la oración» y supone un conocimiento previo.

Más recientemente Sedano ${ }^{13}$ realizó un estudio exhaustivo del verbo ser en oraciones pseudohendidas y las que ella llama oraciones con verbo ser focalizador, como en (6):

(6) Tú estás aquí es por la niña.

Los estudios de este último tipo de oraciones son escasos, debido a que su uso parece estar restringido a algunos países de América, como Colombia, Ecuador, Panamá y Venezuela. De las oraciones escindidas, con la estructura canónica propuesta por Lehmann o las estructuras propuestas por Fernández, no hay estudios en el español de Centroamérica.

\section{Escisiones en el español de Centroamérica}

Los resultados acerca de la estructura de las oraciones escindidas utilizadas por los hablantes en Centroamérica se muestran en el Cuadro 1. Los datos se han calculado a partir de la estructura canónica propuesta por Lehmann (7a) y la propuesta por Fernández (7b).

12 Rodrigo Gutiérrez, «La identificación de los tópicos y los focos», Nueva Revista de Filología Hispánica LVI (2) (2008): 363-401.

13 Mercedes Sedano, «El verbo ser en las oraciones pseudoescindidas y con verbo ser focalizador», Nueva Revista de Filología Hispánica 58 (2010): 39-58. 
(7a) Fue María la que se desmayó.

(7b) María fue la que se desmayó.

En el cuadro 1, en la columna izquierda se ubican las variantes correspondientes a la estructura de las oraciones escindidas; en la columna central, la distribución de las oraciones en relación con el número de hablantes que utiliza cada una. La distribución porcentual se indica en la tercera columna.

Cuadro 1: Estructura de las oraciones escindidas

\begin{tabular}{|l|c||c|}
\hline \multicolumn{1}{|c|}{ Estructura } & $\begin{array}{c}\text { Número de estructuras } \\
\text { escindidas }\end{array}$ & $\mathbf{\%}$ \\
\hline Canónica & 18 & 29,50 \\
\hline Alterna & 43 & 70,50 \\
\hline Total & 61 & $100 \%$ \\
\hline
\end{tabular}

De las sesenta y una oraciones escindidas encontradas durante las veintisiete horas de grabación, aparecen dieciocho con la estructura canónica descrita por Lehmann y cuarenta y tres con la estructura alterna propuesta por Fernández, como se ve en (8) y (9), respectivamente:

(8) Es la familia la que siempre está cerca. (ES-H-1)

(9) La esposa fue la que sufrió mi alcoholismo. (GT-H3)

Según la muestra, en el español de Centroamérica es más habitual utilizar el tópico antes del verbo ser y luego la cláusula relativa; es decir, la estructura que Fernández califica como «improductiva» es más bien la canónica. Por lo general, se parte del supuesto de que los argumentos principales extraídos para formar oraciones escindidas son el sujeto y el objeto. En el cuadro 2, se presentan los resultados de la presente investigación ${ }^{14}$ :

14 Las siglas utilizadas en el cuadro 2, y subsiguientes, son: S: sujeto; O: objeto; DA: dativo; Adv: adverbio; GT: Guatemala; ES: El Salvador; HN: Honduras; NI: Nicaragua; CR: Costa Rica; y PA: Panamá. 


\section{Cuadro 2: Estatus sintáctico del elemento escindido}

\begin{tabular}{|l|c|c|c|c|}
\hline & \multicolumn{1}{l|}{ S } & O & DA & ADV \\
\hline GT & 9 & 3 & - & - \\
\hline ES & 4 & - & - & 13 \\
\hline HN & 2 & 3 & - & 3 \\
\hline NI & 2 & - & 1 & 7 \\
\hline CR & - & 2 & - & 4 \\
\hline PA & 3 & - & - & 5 \\
\hline
\end{tabular}

Estos datos muestran que, con excepción de Guatemala, en Centroamérica el adverbio es el estatus sintáctico mayoritariamente extraído, con un $52,45 \%$. Además, una vez analizados los contextos, se demuestras la función de reiterar y enfatizar, tal como lo han señalado distintos autores. Esto se evidencia en el ejemplo (10), respuesta de la informante a la pregunta de cuál había sido su mayor reto en la vida. Explica que fue cuando ocurrieron los terremotos en 2001, y la llamaron a trabajar como voluntaria en un comité del Programa de Naciones Unidas:

(10) Entonces el programa de Nacional Unidas, dijo, «No. Esta niña tiene madera, vedá; que se quede con nosotros». Pero aparte eeeh esta persona que se llama Lilliam Chiquío, que era la Comisionada Nacional en ese entonces, de la Asociación de Guías, dijo, «No, o sea, yo quiero que también esté en representación de las guías». Y fue así que yo entré al Comité Directivo de la Iniciativa Nacional del Voluntariado. (ES-M-2)

No se suele hacerse una distinción entre extracción de argumentos y de modalidad. Los diferentes estudios se han centrado en los argumentos cuando es evidente que también muchas veces la modalidad 
es la que mayormente se extrae. Tal como lo manifiesta Lambrecht (1994), los hablantes no crean nuevas estructuras para expresar nuevos significados, sino que hacen uso creativo de las estructuras ya existentes de acuerdo con sus intenciones comunicativas, en el caso de esta investigación queda claro que la intención es enfatizar.

\section{Función en la parte de la escindida}

En toda estructura escindida, el constituyente que tiene la oración de relativo sirve para describir, mientras que el otro constituyente (el escindido) es el que se emplea en función referencial. Lambrecht señala que en la función referencial (a la izquierda) se da énfasis e información nueva y la oración de relativo (a la derecha) debe ser presupuesta o existir una acomodación pragmática, tal como se ejemplifica en (11):
(11) Estas son
/ las llaves que perdí.
(información nueva) / (presuposición)

En esta oración el hablante presupone del oyente el conocimiento de que perdió algo y afirma que lo perdido son sus llaves; pero en otras ocasiones no se puede asumir como un hecho conocido del oyente lo expresado en la cláusula relativa, sin embargo la oración no llega a ser inaceptable porque el oyente acomoda el sentido, como en (12).

(12) Hablante: ¿cuándo se lava usted las manos?

Oyente: después de comer (en esta respuesta se acomoda el sentido de que se lava las manos después de comer)

En el cuadro 3 se presenta la función referencial (a la izquierda), que demuestra que en todos los casos los hablantes enfatizan y no hay en ningún ejemplo información nueva; ello se verifica al 
analizar la conversación de cada hablante y se manifiesta que cuando comenta algún tema, a la hora de concluirlo o resumirlo, utiliza la oración escindida, es decir, el hablante enfatiza, no da información nueva. En el cuadro 4 se presenta el estatus morfológico del elemento extraído. La función a la derecha no se analiza pues en todos los casos hay presuposición.

Cuadro 3: Función de la escindida

\begin{tabular}{|l|c|c|c|c|}
\hline & $\begin{array}{c}\text { Total de } \\
\text { oraciones } \\
\text { escindidas }\end{array}$ & $\begin{array}{c}\text { Énfasis } \\
\text { a la } \\
\text { izquierda }\end{array}$ & $\begin{array}{c}\text { Información } \\
\text { nueva a la } \\
\text { izquierda }\end{array}$ & $\%$ \\
\hline GT & 12 & 12 & - & $100 \%$ \\
\hline ES & 17 & 17 & - & $100 \%$ \\
\hline HN & 8 & 8 & - & $100 \%$ \\
\hline NI & 10 & 10 & - & $100 \%$ \\
\hline CR & 6 & 6 & - & $100 \%$ \\
\hline PA & 8 & 8 & - & $100 \%$ \\
\hline
\end{tabular}

Si partimos de lo dicho por los diferentes estudiosos en cuanto que en las oraciones escindidas el foco coincide con información nueva, queda claro (cuadro 3) que en las oraciones analizadas el elemento extraído no se comporta como foco sino como tópico, porque en ninguno de los casos hubo información nueva, sino reiteración para enfatizar. 


\section{Cuadro 4: Estatus morfológico del elemento escindido}

\begin{tabular}{|l|c|c|c|}
\hline & $\begin{array}{c}\text { Total de sujetos } \\
\text { extraídos }\end{array}$ & $\begin{array}{c}\text { Sujeto extraído } \\
\text { pronombre }\end{array}$ & $\begin{array}{c}\text { Sujeto extraído } \\
\text { argumento }\end{array}$ \\
\hline GT & 9 & 6 & 3 \\
\hline ES & 4 & 2 & 2 \\
\hline HN & 2 & 2 & - \\
\hline NI & 2 & 2 & - \\
\hline CR & - & - & - \\
\hline PA & 3 & 3 & - \\
\hline
\end{tabular}

El 52,45\% de las oraciones escindidas extraen el adverbio; el $32,78 \%$ extrae un sujeto. El otro porcentaje es mucho menor $(14,77$ $\%)$ y corresponde a objetos y dativo.

Conforme al cuadro 4, la gran mayoría de los sujetos extraídos (unos 21) corresponde con un pronombre $(71,42 \%)$, el cual enfatiza y resume lo que se venía hablando; es decir, el elemento extraído es topical. En todas las oraciones, sea cual sea el estatus morfológico de lo escindido, en el elemento a la izquierda no se da información nueva, sino que se enfatiza, como se comprueba en (13), en que el informante explica lo que más le gusta hacer:

(13) Bueno eeeh ¿qué me gusta hacer? Eeeh muchas cosas // eeeh pues estar en la universidad, la carrera que estudio, que es sociología, eeeh // de alguna manera con mis otros intereses, conjugarla como mis otros intereses la danza, la música eeeh soy bailarín y también con los scout, soy scout eeeh... y de alguna manera todas esas cosas, pues eeeh mezclarlas ¿verdad? $\mathrm{Y}$ aprender y conjugar esto/todas esas cosas en las actividades que hago, eso es lo que más me gusta hacer. 


\section{Conclusiones}

El español centroamericano presenta varias características propias en relación con las oraciones escindidas. La primera de ellas es la relacionada con la estructura, pues queda evidencia de que la canónica no es la expuesta por Lehmann, ni la que han estudiado otros autores como la suramericana, sino la llamada alternativa. También se muestra que el elemento extraído mayoritariamente es el adverbio, a diferencia de lo manifestado por diversos estudios de que es el sujeto u objeto. Por último, cualquiera que sea el elemento sintáctico que se extraiga, la función principal del elemento a la izquierda es de tópico, pues se reitera para enfatizar y para dar información nueva. 